\title{
Prevalencia del Trastorno por Estrés Postraumático TEPT y eventos asociados en víctimas del desplazamiento forzado en la ciudad de Montería ${ }^{15}$
}

\author{
Kattia Cabas-Hoyos \\ Doctora en Psicología con orientación \\ en neurociencia cognitiva aplicada \\ Universidad del Magdalena. Santa Marta, Colombia \\ Correo electrónico: kcabas@unimagdalena edu.co; \\ kattia.cabas@gmail.com \\ Ilse Villamil Benítez \\ Magíster en Psicología \\ Universidad Pontificia Bolivariana, Colombia \\ Correo electrónico: Ilse.villamil@upb.edu.co
}

\author{
Alicia Uribe Urzola \\ Magíster en Psicología \\ Universidad Pontificia Bolivariana, Colombia \\ Correo electrónico: alicia.uribe@upb.edu.co \\ Carmen Rosa Otero S. \\ Magíster en Psicología \\ Universidad Pontificia Bolivariana, Colombia \\ Correo electrónico: carmen.oteros@upb.edu.co
}

\section{Yaninis González Bracamonte}

Psicóloga

Universidad Pontificia Bolivariana, Colombia Correo electrónico: gonzalez.yaninis@gmail.com
Recibido: 20/05/2020

Evaluado: 02/03/2021

Aceptado: 10/03/2021

\section{Resumen}

El desplazamiento forzoso en Montería puede convertirse en un factor de riesgo para la existencia de Trastorno por Estrés Postraumático (TEPT), sin embargo, no existen datos de prevalencia del mismo. El objetivo de la investigación fue identificar la prevalencia del TEPT y eventos asociados en adultos que sufrieron desplazamiento y están reasentados en esta ciudad. Participaron 117 adultos ( $n=72$ mujeres; $\mathrm{n}=45$ hombres) víctimas del desplazamiento. Para evaluar los eventos asociados al trauma fue empleada la Clinician Administred PTSD Scale CAPS y el PTSD Checklist PCL-C. Se analizaron eventos ocurridos y presenciados por los individuos. Los resultados mostraron que el $26.49 \%$ de la muestra cumplió criterios clínicos de TEPT. Los resultados son consistentes con la literatura existente y establecen un punto de referencia para futuros estudios, así como para el planeamiento de proyectos e intervenciones focalizados en la salud mental de esta población.

15 Para citar este artículo: Cabas-Hoyos, K., Villamil-Benítez, I., Uribe-Urzola, A. Otero, C.R. y GonzálezBracamonte, Y. (2022). Prevalencia del Trastorno por Estrés Postraumático TEPT y eventos asociados en víctimas del desplazamiento forzado en la ciudad de Montería. Informes Psicológicos, 22(1), pp. 251-265. http://dx.doi.org/10.18566/infpsic.v22n1a15 


\section{Prevalence of Post-Traumatic Stress Disorder PTSD and associated events in victims of forced displacement in the city of Monteríavictims of forced displacement in the city of Montería}

Abstract

Forced displacement in Montería can become a risk factor for the existence of Post-Traumatic Stress Disorder (PTSD); however, there are no data on its prevalence. The objective of the research was to identify the prevalence of PTSD and associated events in adults who suffered displacement and are resettled in this city. 117 adults ( $n=72$ women; $n=45$ men) who were victims of displacement participated. To evaluate the events associated with trauma, the Clinician Administered PTSD Scale CAPS and the PTSD Checklist PCL-C were used. Events occurred and witnessed by individuals were analyzed. The results showed that $26.49 \%$ of the sample met clinical criteria for PTSD. The results are consistent with the existing literature and establish a point of reference for future studies, as well as for the planning of projects and interventions focused on the mental health of this population.

\section{Keywords}

Forced displacement, traumatic events, prevalence, PTSD.

\section{Prevalência de transtorno de estresse pós- traumático TEPT e eventos associados em vítimas de deslocamento forçado na cidade de Montería}

Resumo

0 deslocamento forçado em Montería pode se tornar um fator de risco para a existência do Transtorno de Estresse Pós-Traumático (TEPT), porém, não há dados sobre sua prevalência. 0 objetivo da pesquisa foi identificar a prevalência de TEPT e eventos associados em adultos que sofreram deslocamento e são reassentados nesta cidade. Participaram 117 adultos ( $n=72$ mulheres; $n=45$ homens) vítimas de deslocamento. Para avaliar os eventos associados ao trauma, foram utilizados o Clinician Administered PTSD Scale CAPS e o PTSD Checklist PCL-C. Os eventos ocorridos e testemunhados por indivíduos foram analisados. Os resultados mostraram que $026,49 \%$ da amostra preencheu os critérios clínicos para TEPT. Os resultados são consistentes com a literatura existente e estabelecem uma referência para estudos futuros, bem como para o planejamento de projetos e intervenções voltadas para a saúde mental dessa população. 


\section{ntroducción}

El Trastorno de Estrés Postraumático (TEPT) se caracteriza por una sintomatología que se desarrolla después de la exposición directa, observación, conocimiento de algún acontecimiento traumático o exposición repetida o extrema a detalles repulsivos del evento(s) traumático(s) (Clausen et al., 2020; Cloitre, 2020). Este trastorno supone una quiebra profunda en los sentimientos de seguridad personal donde el individuo experimenta sueños angustiantes y recurrentes, malestar psicológico intenso y prolongado, evitación o esfuerzos para evitar los recuerdos, pensamientos o sentimientos estrechamente asociados al suceso(s) traumático(s), alteraciones cognitivas y del estado de ánimo, así como una importante alteración del sistema de alerta en un periodo de cuatro semanas o más (American Psychiatric Association [APA], 2013; Gillbar, Gnall Cole, \& Taft, 2020; Rojas-Campos, 2016).

desplazamiento forzado, por su parte, implica la migración de individuos, familias y comunidades enteras de sus hogares y tierras, siendo una de las consecuencias psicológicamente más devastadoras de la persecución, el conflicto armado, la violencia generalizada y otros tipos de violaciones de los derechos humanos. Esto combinado con los factores estresantes de un trauma y con una pérdida devastadora, aumenta la probabilidad de sintomatologías no sólo del TEPT, sino de trastornos mentales comunes (DMC) como trastorno de depresión mayor, trastorno de ansiedad generalizada, así como quejas somáticas y un mayor consumo de alcohol y drogas ilícitas (Roberts \& Browne, 2011; Shultz et al., 2014).

En Colombia, el desplazamiento forzado a causa de la violencia es un fenómeno de gran magnitud, no sólo ha implicado el despojo abrupto de hogares, negocios, propiedades, activos y posesiones personales, también la pérdida de identidad como terratenientes productivos o ciudadanos con empleo remunerado, estatus social, y redes de apoyo se han visto afectados. En el país, a menudo el proceso de desplazamiento se ha caracterizado por actos de violencia extrema, como amenazas, asaltos violentos, asesinatos, masacres, actos violentos contra líderes cívicos, tortura, secuestro, desaparición forzada, asalto sexual y reclutamiento forzado de jóvenes para servir en grupos al margen de la ley (Albuja et al., 2014; Campo-Arias, Oviedo \& Herazo, 2014; Habozit \& Moro, 2013).

Montería es una ciudad del Caribe Colombiano y capital del Departamento de Córdoba. Este territorio cobra relevancia en la comprensión de la violencia política en Colombia ya que ha padecido el conflicto armado con diversos grupos presentes en la zona. Se plantea que desde 1985 empezó a llegar un número importante de personas desplazadas a la región y ya hacia el año 2003 habían existido unas 30 invasiones donde vivían desplazados (Negrete, 2008). De acuerdo con cifras reveladas por el Registro Único de Víctimas (RUV, 2020), el número de personas registradas en el país como víctimas de desplazamiento forzado es de 8.011.693. En la categoría de hechos victimizantes, Córdoba reporta un acumulado de 301.083 víctimas de desplazamiento forzado y 4.621 por desaparición forzada. Montería, por su parte, cuenta 
con 90.356 víctimas de desplazamiento forzado y 1.673 de desaparición forzada.

Según Shultz et al. (2014), el proceso de desplazamiento de Colombia difiere de otros en el mundo debido a que es un proceso prolongado que implica experiencias de vida donde se destacan: 1) amenazas previas a la expulsión y vulnerabilidad en la comunidad de origen; 2) eventos precipitantes que incluyen el mismo momento del desplazamiento; 3) la migración en búsqueda de un hábitat más seguro; 4) la transición durante la reubicación inicial; 5) el reasentamiento a largo plazo; y 6) el retorno a la comunidad de origen.

El presente estudio tendrá como foco la fase de reasentamiento por ser un proceso que persiste durante décadas y que se constituye como el "Nuevo Normal" de quienes lo padecen. Los desplazados reasentados se enfrentan a modificaciones en todos los aspectos de la vida y la calidad de ésta, a saber, aprender a hacer frente a los sistemas urbanos, ingresos escasos en los trabajos del sector informal, buscar apoyo de diversos servicios sociales, y lidiar con los factores estresantes de la violencia urbana generalizada (Richards et al., 2014; Shultz et al., 2014).

Esta investigación se considera pertinente debido a que se desconocen datos estadísticos que den a conocer sobre las características de la salud mental en víctimas del desplazamiento de la región y se proyecta como un primer paso para considerar el planteamiento de futuras intervenciones psicológicas eficaces. Se hace necesario generar este tipo de estudios de manera que permita establecer perfiles propios de los desplazados de una zona del país para identificar lo que pueden tener en común o bien lo que lo podría distinguirlos de los refugiados de resto del mundo, así como para plantear intervenciones psicológicas eficaces para este colectivo.

El presente estudio tiene como objetivo estimar la prevalencia del TEPT y los factores asociados en una población víctima del desplazamiento forzoso y que actualmente se encuentran reasentados en la Ciudad de Montería, departamento de Córdoba. Se hipotetiza un nivel considerable de prevalencia en la población objeto.

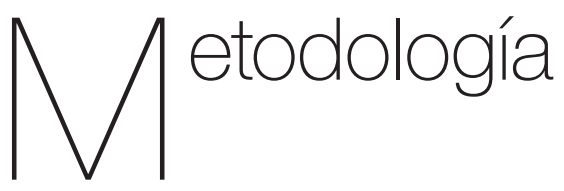

\section{Diseño}

El presente estudio es transversal porque logra describir las variables y analizar su incidencia en un momento único de tiempo. Así mismo, cuenta con un diseño no experimental debido a que no se manipularon las variables (Hernández-Sampieri, Fernández-Collado \& Baptista-Lucio, 2010). El enfoque de la presente investigación es cuantitativo dado que recolecta, analiza y vincula datos de tipo numérico, no obstante se vale de técnicas propias de la evaluación clínica para dar respuesta a la pregunta de investigación; su alcance es exploratorio puesto que investiga un problema poco estudiado, ayudando así a identificar conceptos promisorios.

\section{Participantes}

La muestra final estuvo conformada por 117 adultos de los cuales el 38.46\% ( $n=45$ ) fueron hombres y el 61.53\% ( $n=72$ ) fueron mujeres, con edades 
comprendidas entre 18 y 71 años. La media de edad fue de 40.41 años (DT = 13.14). Los niveles de escolaridad que más predominaron fueron secundaria con $47 \%$ ( $n=$ 55), primaria con $41.02 \%(n=48)$ y, en menor proporción, formación técnica (4.1\%; n $=5$ ). Todos los sujetos fueron informados acerca del estudio, firmaron consentimiento informado y participaron voluntariamente sin recibir pago por su participación; por último, se les informó que podían retirarse en cualquier momento.

Los participantes fueron seleccionados a través de un muestreo intencional. Se tomó, para ello, el 12\% de la población censada por el Programa de Ciudadanía de la Alcaldía de Montería en el año 2017, cumpliendo con los requisitos de etapa de reasentamiento de Shultz et al. (2014).

\section{Instrumentos}

Instrumento para valoración de TEPT: Clinician-Administered PTSD Scale (CAPS) (Blake et al., 1990). Es una entrevista estructurada que contiene 30 ítems y evalúa 17 síntomas sobre TEPT según el DSM-IV. Incluye además 8 ítems que evalúan sentimientos de culpabilidad y factores asociados e igualmente se divide en tres subescalas que corresponden a criterios clínicos para el diagnóstico, es decir, reexperimentación, evitación e hiperactivación. La versión adaptada en población mexicana ( $\mathrm{Pa}$ lacios, 2002) tiene un Alfa de Cronbach = .85. El punto de corte establecido es 40 en la escala global para lograr el diagnóstico. Así mismo, permiten identificar los eventos traumáticos que el sujeto ha experimentado. Para fines de este estudio se analizaron estos eventos en dos categorías: "me sucedió a mi" y "lo presencié", no incluyendo la categoría "supe que le ocurrió a alguien".
Esta última no se incluyó debido a que los autores consideraron "me sucedió a mi" y "lo presencié" se consideraban factores suficientes para explicar el diagnóstico de TEPT.

Lista de chequeo para estrés postraumático TEPT. EI PTSD Checklist PCL-C es una versión corta del PTSD Checklist - Civilian version, desarrollado por Weathers, Litz, Herman, Huska \& Keane (1993). Evalúa la sintomatología del TEPT en una población vulnerable tras sufrir eventos adversos. Consta de 17 ítems que hacen referencia a problemas y molestias que podrían experimentarse en situaciones generadoras de estrés. Los eventos se distribuyen en tres factores: intrusión, evitación y activación, según la clasificación establecida en el DSM-IV-TR (APA, 2000). Este instrumento fue traducido y adaptado del inglés al español por un grupo de expertos en población mexicana, donde posteriormente se determinó su validez y confiabilidad. El Alfa de Cronbach total del instrumento fue de .92 (Flores-Morales, Reyes-Pérez \& Reidl-Martínez, 2012).

\section{Setting}

La investigación fue conducida por psicólogos clínicos entrenados para tal fin y se llevó a cabo en el salón comunitario de las urbanizaciones. La evaluación se realizó en una sesión de 90 minutos por participante.

\section{Análisis de datos}

Fueron empleados estadísticos descriptivos sobre los tipos de eventos vivenciados por los individuos; posteriormente, se realizó una comparación mediante 
pruebas T para muestras independientes para comparar los factores asociados al TEPT por categorías (la significación estadística estimada fue del .05). El tratamiento de los datos se realizó mediante SPSS 24.0 versión castellano.

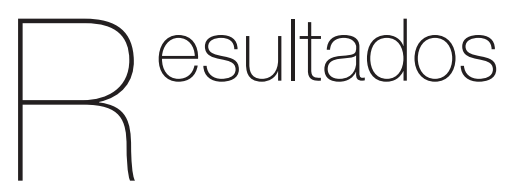

Para dar cumplimiento al objetivo de la investigación, se indagó sobre los sucesos traumáticos que propician la sintomatología relacionada con traumas y que ponen en riesgo el bienestar psicológico (ver Tabla 1). Los eventos más prevalentes referidos por los sujetos en la categoría "me sucedió mí" fueron: desastre natural en un $42.70 \%$ $(n=50) ;$ muerte inesperada de alguien cercano (35.90\%; $n=42)$, y sufrimiento humano grave, con un $27.40 \%(n=32)$. En la categoría "lo presencié" los sucesos de mayor frecuencia fueron: muerte inesperada de alguien cercano en un 19.0\% ( $n=$ 23); zonas de combate y accidente de tráfico/transporte obtuvieron niveles similares, a saber, $15.40 \%$ ( $n=18$ ); y, por último, se encontró que desastre natural tuvo una frecuencia de $14.5 \%(n=17)$.

Tabla 1.

Frecuencia de eventos vitales traumáticos

\begin{tabular}{|c|c|c|c|c|c|c|}
\hline & \multicolumn{2}{|c|}{ “Me sucedió a mi” } & \multicolumn{2}{|c|}{ "Lo Presencié” } & \multicolumn{2}{|c|}{ Total } \\
\hline & $\mathrm{N}$ & Porcentaje & $N$ & Porcentaje & $\mathrm{N}$ & Porcentaje \\
\hline Desastre Natural & 50 & $42.7 \%$ & 17 & $14.5 \%$ & 67 & $57.26 \%$ \\
\hline Incendio Explosión & 10 & $8.5 \%$ & 15 & $12.8 \%$ & 25 & $21.36 \%$ \\
\hline Accidente Transporte & 19 & $16.2 \%$ & 18 & $15.4 \%$ & 37 & $31.60 \%$ \\
\hline Accidente Grave otros & 17 & $14.5 \%$ & 10 & $8.5 \%$ & 27 & $23.10 \%$ \\
\hline Sustancias tóxicas & 5 & $4.3 \%$ & 8 & $6.8 \%$ & 13 & $11.1 \%$ \\
\hline Agresión física & 22 & $18.8 \%$ & 7 & $6.0 \%$ & 29 & $24.8 \%$ \\
\hline Agresión arma & 18 & $15.4 \%$ & 12 & $10.3 \%$ & 30 & $25.6 \%$ \\
\hline Agresión sexual & 14 & $12.0 \%$ & 2 & $1.7 \%$ & 16 & $13.5 \%$ \\
\hline Experiencia sexual incómoda & 6 & $5.1 \%$ & 3 & $2.6 \%$ & 9 & $7.7 \%$ \\
\hline Zona combate & 26 & $22.2 \%$ & 18 & $15.4 \%$ & 44 & $37.6 \%$ \\
\hline Cautiverio & 5 & $4.3 \%$ & 1 & $.9 \%$ & 6 & $5.1 \%$ \\
\hline Enfermedad lesión riesgo & 14 & $12 \%$ & 3 & $2.6 \%$ & 17 & $14.5 \%$ \\
\hline Sufrimiento humano grave & 32 & $27.4 \%$ & 8 & $6.8 \%$ & 40 & $34.2 \%$ \\
\hline Muerte repentina violenta & 14 & $12.0 \%$ & 16 & $13.7 \%$ & 30 & $25.6 \%$ \\
\hline Muerte inesperada cercana & 42 & $35.9 \%$ & 23 & $19.7 \%$ & 65 & $55.6 \%$ \\
\hline Lesiones graves daños & 3 & $2.6 \%$ & 4 & $3.4 \%$ & 7 & $6.0 \%$ \\
\hline Maltrato/abuso sexual en la infancia & 23 & $19.7 \%$ & 5 & $4.3 \%$ & 28 & $23.93 \%$ \\
\hline
\end{tabular}

Fuente: autores. 
Fueron contrastados los eventos de la Tabla 1 en relación a la presencia/ausencia de TEPT (ver Tabla 2) encontrándose que quienes cumplieron criterios para el trastorno indicaron la existencia de eventos como: muerte repentina de un familiar cercano $(n=20)$, sufrimiento humano grave $(n=19)$ y desastre natural $(n=19)$ en mayores proporciones; entre tanto, quienes no cumplieron con criterios para TEPT, presentaron mayores niveles en eventos como: desastre natural $(n=48)$, muerte repentina de un familiar cercano $(n=45)$ y exposición a zonas de combate $(n=27)$.

Tabla 2.

Estadísticos descriptivos de eventos traumáticos en relación a presencia/ausencia de TEPT

\begin{tabular}{|c|c|c|c|}
\hline & $\mathrm{N}$ & $M(D . T)^{\star}$ & Error típ. de la M \\
\hline & $P / A^{*}$ & $\mathrm{P} / \mathrm{A}$ & $\mathrm{P} / \mathrm{A}$ \\
\hline Desastre Natural & $19 / 48$ & $1.26(.452) / 1.25(.438)$ & $.104 / .063$ \\
\hline Incendio Explosión & $11 / 14$ & $1.36(.505) / 1.79(.426)$ & $.152 / .114$ \\
\hline Accidente Transporte & $18 / 19$ & $1.44(.511) / 1.53(.513)$ & $.121 / .118$ \\
\hline Accidente Grave otros & $10 / 17$ & $1.30(.483) / 1.41(.507)$ & $.153 / .123$ \\
\hline Sustancias tóxicas & $4 / 9$ & $1.25(.500) / 1.78(.441)$ & $.250 / .147$ \\
\hline Agresión física & $14 / 15$ & $1.14(.363) / 1.33(.488)$ & $.097 / .126$ \\
\hline Agresión arma & $12 / 18$ & $1.33(.492) / 1.44(.511)$ & $.142 / .121$ \\
\hline Agresión sexual & $9 / 7$ & $1.22(.441) / 1.00(.000)$ & $.147 / .000$ \\
\hline Experiencia sexual incómoda & $5 / 4$ & $1.00(.000) / 1.75(.500)$ & $.000 / .250$ \\
\hline Zona combate & $17 / 27$ & $1.41(.507) / 1.41(.501)$ & $.123 / .096$ \\
\hline Cautiverio & $4 / 2$ & $1.25(.500) / 1.00(.000)$ & $.250 / .000$ \\
\hline Enfermedad lesión riesgo & $11 / 6$ & $1.00(.000) / 1.50(.548)$ & $.000 / .224$ \\
\hline Sufrimiento humano grave & $19 / 21$ & $1.16(.375) / 1.24(.436)$ & $.086 / .095$ \\
\hline Muerte repentina violenta & $9 / 21$ & $1.78(.441) / 1.43(.507)$ & $.147 / .111$ \\
\hline Muerte repentina cercana & $20 / 45$ & $1.40(.503) / 1.33(.477)$ & $.112 / .071$ \\
\hline Lesiones graves daños & $1 / 6$ & $2.00(.) / 1.50(.548)$ & . \\
\hline Maltrato abuso infancia & $13 / 16$ & $1.15(.376) / 1.38(.806)$ & $.224 / .202$ \\
\hline
\end{tabular}

Nota: M (D.T)= Media (Desviación Típica). P/A= Presencia/Ausencia. Fuente: autores

Se comparó la ocurrencia de estos eventos en relación al sexo (ver Tabla 3). Las mujeres refirieron, en mayor medida, desastres naturales $(n=44)$, muerte repentina de una persona cercana $(\mathrm{n}=$ 40) y exposición a zonas de combate (n = 33); mientras que los hombres, por su parte, reportaron eventos relacionados 
con muerte repentina de una persona cercana $(n=25)$, desastre natural $(n=$ 23) y sufrimiento humano grave $(n=16)$.
Estos últimos refirieron menor número de eventos traumáticos comparado con las participantes.

Tabla 3.

Estadísticos descriptivos de eventos traumáticos en relación al sexo.

\begin{tabular}{|c|c|c|c|}
\hline & $\begin{array}{c}\mathrm{N} \\
\mathrm{M} / \mathrm{H}^{*}\end{array}$ & $\begin{array}{c}M(D . T) * \\
M / H\end{array}$ & $\begin{array}{c}\text { Error típ. De la M } \\
M / H^{*}\end{array}$ \\
\hline Desastre Natural & $44 / 23$ & $1.30(.462) / 1.17(.388)$ & $.070 / .081$ \\
\hline Incendio Explosión & $15 / 10$ & $1.73(.458) / 1.40(.516)$ & $.118 / .163$ \\
\hline Accidente Transporte & $24 / 13$ & $1.54(.509) / 1.38(.506)$ & $.104 / .140$ \\
\hline Accidente Grave otros & $18 / 9$ & $1.44(.511) / 1.22(.441)$ & $.121 / .147$ \\
\hline Sustancias tóxicas & $6 / 7$ & $1.83(.408) / 1.43(.535)$ & $.167 / .202$ \\
\hline Agresión física & $19 / 10$ & $1.26(.452) / 1.20(.422)$ & $.104 / .133$ \\
\hline Agresión arma & $19 / 11$ & $1.42(.507) / 1.36(.505)$ & $.116 / .152$ \\
\hline Agresión sexual & $6 / 10$ & $1.33(.516) / 1.00(.000)$ & $.211 / .000$ \\
\hline Experiencia sexual incómoda & $5 / 4$ & $1.40(.548) / 1.25(.500)$ & $.245 / .250$ \\
\hline Zona combate & $33 / 11$ & $1.39(.496) / 1.45(.522)$ & $.086 / .157$ \\
\hline Cautiverio & $3 / 3$ & $1.33(.577) / 1.00(.000)$ & $.333 / .000$ \\
\hline Enfermedad lesión riesgo & $9 / 8$ & $1.33(.577) / 1.00(.000)$ & $.167 / .000$ \\
\hline Sufrimiento humano grave & $24 / 16$ & $1.25(.442) / 1.13(.342)$ & $.090 / .085$ \\
\hline Muerte repentina violenta & $18 / 12$ & $1.44(.511) / 1.67(.492)$ & $.121 / .142$ \\
\hline Muerte repentina cercana & $40 / 25$ & $1.33(.474) / 1.40(.500)$ & $.075 / .100$ \\
\hline Lesiones graves daños & $5 / 2$ & $1.60(.548) / 1.50(.707)$ & $.245 / .500$ \\
\hline Maltrato abuso infancia & $19 / 10$ & $1.16(.375) / 1.50(.972)$ & $.086 / .307$ \\
\hline
\end{tabular}

Nota: M (D.T) = Media (Desviación Típica). M/H = Mujer/Hombre. Fuente: autores

Así mismo, se estableció una comparación por grupos etarios (ver Tabla 4). Los participantes más jóvenes indicaron, en mayor medida, desastres naturales ( $\mathrm{n}$ = 31), muerte repentina de una persona cercana $(n=24)$ y exposición a zonas de combate $(n=15)$. Entre tanto, aquellos con una tasa etaria mayor refirieron eventos como: muerte repentina de una persona cercana $(n=41)$, desastre natural $(n=36)$ y zona de combate $(n=29)$. La tendencia indica que los participantes mayores fueron quienes más vivenciaron algún evento traumático. No obstante, no fue posible calcular si tales diferencias resultaron estadísticamente significativas, dada la variabilidad de eventos traumáticos referidos. 
Tabla 4.

Estadísticos descriptivos de eventos traumáticos en relación al grupo etario

\begin{tabular}{lccc}
\hline & $\begin{array}{c}\mathbf{N} \\
\mathbf{J} / \mathbf{M}^{*}\end{array}$ & $\begin{array}{c}\mathbf{M}(\mathbf{D . T})^{\star} \\
\mathbf{J} / \mathbf{M}\end{array}$ & $\begin{array}{c}\text { Error típ. de la M } \\
\text { J/M }\end{array}$ \\
\hline Desastre Natural & $31 / 36$ & $1.16(.374) / 1.33(.478)$ & $.067 / .080$ \\
Incendio Explosión & $6 / 19$ & $1.83(.408) / 1.53(.513)$ & $.167 / .118$ \\
Accidente Transporte & $10 / 27$ & $1.40(.516) / 1.52(.509)$ & $.163 / .098$ \\
Accidente Grave otros & $13 / 14$ & $1.31(.480) / 1.43(.514)$ & $.133 / .137$ \\
Sustancias tóxicas & $6 / 7$ & $1.67(.516) / 1.57(.535)$ & $.211 / .202$ \\
Agresión física & $9 / 20$ & $1.33(.500) / 1.20(.410)$ & $.167 / .092$ \\
Agresión arma & $11 / 19$ & $1.55(.522) / 1.32(.478)$ & $.157 / .110$ \\
Agresión sexual & $10 / 6$ & $1.00(.000) / 1.33(.516)$ & $.000 / .211$ \\
Experiencia sexual incómoda & $4 / 5$ & $1.50(.577) / 1.20(.447)$ & $.289 / .200$ \\
Zona combate & $15 / 29$ & $1.53(.516) / 1.34(.484)$ & $.133 / .090$ \\
Cautiverio & $1 / 5$ & $1.00(.) / 1.20(.447)$ &.$/ .200$ \\
Enfermedad lesión riesgo & $3 / 14$ & $1.33(.577) / 1.14(.363)$ & $.333 / .097$ \\
Sufrimiento humano grave & $13 / 27$ & $1.31(.480) / 1.15(.362)$ & $.133 / .070$ \\
Muerte repentina violenta & $11 / 19$ & $1.64(.505) / 1.47(.513)$ & $.152 / .118$ \\
Muerte repentina cercana & $24 / 41$ & $1.38(.495) / 1.34(.480)$ & $.101 / .075$ \\
Lesiones graves daños & $2 / 5$ & $2.00(.000) / 1.40(.548)$ & $.000 / .245$ \\
Maltrato abuso infancia & $13 / 16$ & $1.38(.870) / 1.19(.403)$ & $.241 / .101$ \\
\hline Nota: & & & \\
\hline
\end{tabular}

Nota: M (D.T) = Media (Desviación Típica). J/A = Jóvenes/Mayores.

Fuente: autores

\section{Prevalencia de TEPT}

Se determinó la presencia de TEPT comprobada mediante la lista de Chequeo para TEPT (Weathers et al., 1993)

Tabla 5.

Estadístico descriptivo para Presencia/ausencia de TEPT

\begin{tabular}{rccc}
\hline & $\begin{array}{c}\text { N } \\
\text { P/A }^{*}\end{array}$ & $\begin{array}{c}\text { Porcentaje } \\
\text { P/A }\end{array}$ & $\begin{array}{c}\text { Porcentaje } \\
\text { válido }\end{array}$ \\
\hline Prevalencia de TEPT & $31 / 86$ & $26.5 \% / 73.5 \%$ & $26.5 \% / 73.5 \%$ \\
Total & 117 & 100.0 & 100.0 \\
\hline
\end{tabular}

Nota: $\mathrm{P} / \mathrm{A}=$ Presencia/Ausencia

Fuente: autores

Relacionado al sexo (ver Tabla 6), aunque los niveles de TEPT fueron ligeramente mayores en la muestra masculina, una prueba $T$ para muestras independientes permitió establecer que no hubo y se encontró que el $26.5 \%$ ( $n=31$ ) cumplió criterios de TEPT y el 73.5\% ( $n=86)$ no presentó criterios para el trastorno (ver Tabla 5). 
Tabla 6.

Estadísticos de grupo. Nivel total de TEPT por sexo

\begin{tabular}{llcc} 
& $\mathbf{N}$ & M(D.T) & Error típ. de la $\mathbf{M}$ \\
& M/H* & & \\
\hline Nivel de TEPT & $72 / 45$ & $33.06(14.359) / 35.24(16.137)$ & $1.692 / 2.406$ \\
\hline
\end{tabular}

Nota: $\mathrm{M}(\mathrm{D} . \mathrm{T})=$ Media (Desviación Típica). $\mathrm{M} / \mathrm{H}=$ Mujer/Hombre

Fuente: autores

Teniendo en cuenta que en la lista de chequeo para TEPT fue establecido la activación, intrusión y evitación según el DSM-IV-TR (APA, 2000), se contrastaron estos factores en relación al sexo y a los grupos etarios mediante una prueba $T$ para muestras independientes.

Al contrastar la presencia de TEPT por grupos etarios los niveles fueron significativamente mayores en los individuos de más edad [TEPT $F=(1.117)=$ 1.50; $p=.02$ ]. Sin embargo, los factores mostraron una mayor tendencia en los individuos mayores sin resultar estadísticamente significativos [Activación $\mathrm{F}=(1.117)$ = 1.90; $p=.19$ ] [Intrusión $F=(1.117)=1.50$; $p=.22$ ] [Evitación $F=(1.117)=.50 ; p=.82$ ] (ver Tabla 7).

Tabla 7.

Estadísticos de grupo. Factores asociados a TEPT por grupos etarios

\begin{tabular}{lccc}
\hline & $\begin{array}{c}\mathbf{N} \\
\mathbf{J} / \mathbf{M}^{*}\end{array}$ & $\begin{array}{c}\mathbf{M}(\mathbf{D} . \mathbf{T})^{*} \\
\text { J/M }\end{array}$ & $\begin{array}{c}\text { Error típ. de la M } \\
\text { J/M }\end{array}$ \\
\hline TEPT - Total & $52 / 65$ & $29.21(13.773) / 37.65(5.052)$ & $1.910 / 1.867$ \\
Factor "Activación" & $52 / 65$ & $12.19(6.180) / 16.08(6.890)$ & $.857 / .855$ \\
Factor "Intrusión" & $52 / 65$ & $8.63(4.559) / 10.86(5.417)$ & $.632 / .672$ \\
Factor "Evitación" & $52 / 65$ & $8.38(4.371) / 10.71(4.772)$ & $.606 / .592$ \\
\hline
\end{tabular}

Nota: $\mathrm{M}(\mathrm{D} . \mathrm{T})$ = Media (Desviación Típica). J/A = Jóvenes/Mayores. Fuente: autores

Con relación al factor de activación se encontró que los hombres de la muestra presentaron diferencias significativas frente a las mujeres [Activación. $F=(1.117)=$ .79; $p=0.00]$. Los factores de intrusión y evitación mostraron también ligeras tendencias que no permitieron que se alcanzara la significación estadística [Intrusión. $\mathrm{F}=(1.117)=1.90 ; \mathrm{p}=.16] ;$ [Evitación. $\mathrm{F}=$ $(1.117)=.05 ; p=.80]$ (ver Tabla 8).

Tabla 8.

Estadísticos de grupo. Factores asociados a TEPT distribuídos por sexo

\begin{tabular}{lccc}
\hline & $\begin{array}{c}\text { N } \\
\text { M/H* }\end{array}$ & $\begin{array}{c}\text { M(D.T) } \\
\text { M/H }\end{array}$ & $\begin{array}{c}\text { Error típ. de la M } \\
\text { M/H }\end{array}$ \\
\hline Factor "Activación" & $72 / 45$ & $13.90(6.557) / 15.07(2.281)$ & $.773 / .485$ \\
Factor "Intrusión" & $72 / 45$ & $9.57(4.898) / 10.36(5.560)$ & $.577 / .829$ \\
Factor "Evitación" & $72 / 45$ & $9.58(4.623) / 9.82(4.928)$ & $.545 / .735$ \\
\hline
\end{tabular}

Nota: $\mathrm{M}(\mathrm{D} . \mathrm{T})$ = Media (Desviación Típica). $\mathrm{M} / \mathrm{H}=$ Mujer/Hombre

Fuente: autores 


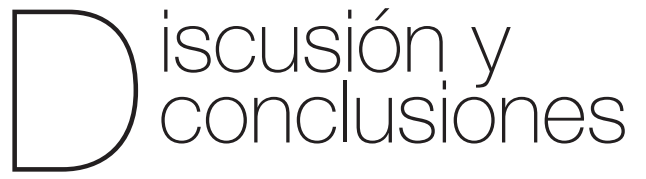

conflicto armado colombiano ha puesto a la población civil como un foco militar donde las luchas sociopolíticas han llevado a que un sinnúmero de personas en condiciones vulnerables sean forzadas a dejar sus pueblos y focalicen las ciudades como zonas de refugio. La violencia como factor transversal suscita la vulnerabilidad y la exposición a eventos que afectan la salud mental, aumentando la necesidad de una atención integral que mejore la calidad de vida de los afectados (Venegas-Luque, Gutiérrez-Velasco \& Calcedo-Cardeñosa, 2017).

No obstante, es preciso señalar que, a pesar de que ha habido esfuerzos en temas de legislación para atender a las víctimas y se ha puesto en marcha la Ley de Víctimas y Restitución de Tierras (Ley 1448 de 2011) (Congreso de Colombia, 2011), éstas no inciden sobre la disminución y mucho menos la desaparición del trauma. Según Venegas-Luque et al. (2017), las acciones de reparación no resultan suficientes para superar efectos psicológicos negativos en las víctimas; secuelas ligadas al desarrollo de trastornos como los del estado de ánimo, de alimentación y, en especial, el TEPT. Ante esta situación, se considera que este tipo de trastornos requieren de una intervención especializada

Se calcula que el TEPT afecta entre $5-10 \%$ de la población (los accidentes y lesiones son los más frecuentes reportados, con un $36 \%$ de prevalencia vitalicia en todo el mundo) y cuya incidencia se refleja en múltiples sistemas biológicos desde los circuitos cerebrales hasta el sistema inmune. En este trastorno, los factores de riesgo psicosociales y biológicos se consideran predictores del transcurso del mismo, aunque el trauma sigue siendo un factor clave como precipitante (Shalev, Liberzon \& Marmar, 2017; Yehuda et al., 2015).

El presente estudio evidencia que los niveles de TEPT en desplazados reasentados son superiores a los encontrados en el estudio de Ramírez-Giraldo, Hernández-Bustamante, Romero-Acosta \& Porras-Mendoza (2017), quienes encuentran en Chengue, Sucre, un nivel del $26.2 \%$. Este último estudio se considera un referente teórico importante debido a las características socioculturales de la muestra y a la proximidad entre los territorios donde fueron desarrollados.

La relación significativa del desplazamiento forzado y vulnerabilidades preexistentes (como adversidades familiares y otros traumas) con la alta prevalencia del TEPT, como indica Bromet et al. (2017), coincide con los resultados obtenidos, teniendo en cuenta el conflicto armado como factor que no causa uno sino múltiples efectos sobre las víctimas del mismo. Adicionalmente se logra evidenciar con el presente estudio que mayores niveles de TEPT estuvieron presentes en los individuos con mayor edad, lo cual estaría explicado por una variedad de experiencias traumáticas vividas, así como diferentes sucesos adversos que se manifiestan en sintomatología subclínica. Otro aspecto a destacar es que en la presente investigación la presencia del TEPT no mostró diferencias significativas en relación al sexo, lo que contradice a la literatura disponible que señala que el TEPT se presenta con mayor frecuencia en mujeres (Kessler et 
al., 2014; Bados, 2015; Torres-Salazar, Mejía-Jaimes, Conde-Cotes \& BotelhoDe Oliveira, 2021).

Los resultados de este estudio concuerdan con lo planteado por Pérez-Sierra, Muñiz-Gil, Jaramillo-Rico y GómezVásquez (2004) quienes afirman que el desplazamiento es un evento determinante en la historia de vida de los individuos y se pueden materializar los efectos arrasadores que producen las pérdidas materiales y la angustia que esto genera. En los desplazados reasentados de Montería se encuentra que la violencia sociopolítica no solo ha tenido un efecto devastador sobre los desplazados, sino una multiplicidad de situaciones por enfrentar que los revictimizan constantemente. Estos eventos que se dan en la sociedad también generan un alto desequilibrio en la salud mental de los seres humanos que en muchas ocasiones desencadena en psicopatologías. De esta manera, es importante considerar el carácter multifactorial del TEPT y su relación con la presencia de eventos traumáticos (Bados, 2015; Kessler et al., 2014). La prevalencia de este trastorno no solo es poco común en países de altos ingresos, sino que está altamente relacionada con la gravedad de la exposición al estrés y los antecedentes previos a esta misma exposición (Bromet et al., 2017).

A pesar de que en este estudio no se encontraron diferencias significativas relacionadas con el sexo y edad de los participantes (lo cual contradice a la literatura disponible), esta investigación aporta datos relevantes que servirían de base para futuros estudios enfocados a una mayor comprensión entre el TEPT relacionado con violencia sociopolítica y factores asociados, así como futuras evaluaciones e intervenciones encaminadas a mejorar la calidad de vida y salud mental de personas víctimas del desplazamiento forzado.

\section{Financiación}

Este es un producto del proyecto denominado: "Tratamiento del Trastorno por Estrés Postraumático (TEPT) en víctimas $y$ testigos de violencia" financiado por el Centro de Investigación para el Desarrollo y la Innovación CIDI de la Universidad Pontificia Bolivariana, Montería.

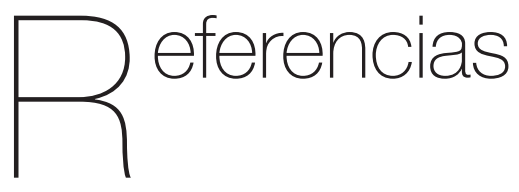

Albuja, S., Arnaud, E., Caterina, M., Charron, G., Foster, F., Glatz, A. K.,... Wissing, M. (2014). Global Overview 2014, people internally displaced by conflict and violence. Retrieved from https://www.internaldisplacement.org/sites/default/files/inlinefiles/201405-global-overview-2014-en.pdf

American Psychiatric Association (2000). Diagnostic and Statistical Manual of Mental Disorders (4th ed.). Washington, DC: American Psychiatric Association.

American Psychiatric Association (2013). Diagnostic and Statistical Manual of Mental Disorders (5th ed.). Washington, DC: Author.

Bados, A. (2015). Trastorno por estrés postraumático. Recuperado de http://diposit.ub.edu/dspace/ bitstream/2445/65623/1/TEPT.pdf 
Blake, D. D., Weathers, F., Nagy, L. M., Kaloupek, D. G., Klauminzer, G., Charney, D. S., \& Keane, T. M. (1990). A clinical rating scale for assessing current and lifetime PTSD: The CAPS-1. Behavior Therapy, 18(18), 187-188. https://doi.org/10.1007/ BF02105408

Bromet, E. J., Atwoli, L., Kawakami, N., Navarro-Mateu, F., Piotrowski, P., King, A. J., ... \& Florescu, S. (2017). Post-traumatic stress disorder associated with natural and human-made disasters in the World Mental Health Surveys. Psychological Medicine, 47(2), 227-241. doi: 10.1017/ S0033291716002026.

Campo-Arias, A., Oviedo, H. C., \& Herazo, E. (2014). Prevalencia de síntomas, posibles casos y trastornos mentales en víctimas de conflicto armado interno en situación de desplazamiento en Colombia: una revisión sistemática. Revista Colombiana de Psiquiatría, 43(4), 177-185. doi: 10.10167j. rcp.2014.07.003

Clausen, A. N., Clarke, E., Phillips, R. D., Haswell, C., VA Mid-Atlantic MIRECC Workgroup., \& Morey, R. A. (2020). Combat exposure, posttraumatic stress disorder, and head injuries differentially relate to alterations in cortical thickness in military Veterans. Neuropsychopharmacology, 45(3), 491498. doi:10.1038/s41386-019-0539-9

Cloitre, M. (2020). ICD-11 complex posttraumatic stress disorder. Simplifying diagnosis in trauma populations. The British Journal of Psychiatry, 216(3), 129131. doi: 10.1192/bjp.2020.43

Congreso de Colombia. (2011). Ley 1448 de 2011. Recuperado de https:// www.unidadvictimas.gov.co/es/ ley-1448-de-2011/13653
Flores-Morales, R., Reyes-Pérez, V., \& ReidlMartínez, L. M. (2012). Síntomas de Estrés Postraumático (EPT) en periodistas mexicanos que cubren la guerra contra el narcotráfico. Suma Psicológica, 19(1), 7-17. Recuperado de: http://www.scielo.org.co/ pdf/sumps/v19n1/v19n1a01.pdf

Gillbar O., Gnall K. E., Cole, H. E., \& Taft, C. T. (2020) Posttraumatic Stress Disorder, Intimate Partner Violence, and TraumaInformed Intervention. In B. Carpiniello, A. Vita, \& C. Mencacci (Eds.), Violence and Mental Disorders. Comprehensive Approach to Psychiatry (pp. 115-134). Cham, Switzerland: Springer. doi: 10.1007/978-3-030-33188-7

Habozit, A., \& Moro, M. R. (2013). Breaking the silence in Colombia to resist violence 1/2. Soins Psychiatrie, 284(1), 34-42. doi: 10.1016/j.spsy.2012.11.006

Hernández-Sampieri, R., Fernández-Collado, C., \& Baptista-Lucio, P. (2010). Metodología de la investigación. México: Editorial Mc Graw Hill.

Kessler, R. C., Rose, S., Koenen, K. C., Karam, E. G., Stang, P. E., Stein, D. J., ... \& McLean, S. A. (2014). How well can post-traumatic stress disorder be predicted from pretrauma risk factors? An exploratory study in the WHO World Mental Health Surveys. World Psychiatry, 13(3), 265-274. doi: 10.1002/wps.20150.

Negrete, V. (2008). Problemática psicosocial y socieconómica como consecuencia del conflicto armado en el departamento de Córdoba en Colombia. International Journal of Psychological Research, 1(1), 7480. https://doi.org/10.21500/20112084.969 
Palacios, L. (2002). Trastorno por estrés postraumático: Factores asociados a la remisión y/o persistencia del diagnóstico. Una evaluación de su curso en pacientes de una muestra mexicana en el Instituto Nacional de Psiquiatría Ramón de la fuente Muñiz (Tesis de especialización). Universidad Nacional Autónoma de México, México D.F.

Pérez-Sierra, M., Muñiz-Gil, O., JaramilloRico, J. \& Gómez-Vásquez, C. (2004). "Desplazamiento sin sentido": estudio clínico de la experiencia violenta y del desplazamiento. Informes Psicológicos, (6), 161-174. Recuperado de: https://dialnet.unirioja.es/servlet/ articulo?codigo $=7051929$

Ramírez-Giraldo, A., Hernández-Bustamante, O., Romero-Acosta, K., \& PorrasMendoza, E. (2017). Estado de salud mental de personas víctimas del conflicto armado en Chengue. Psicología desde el Caribe, 34(1), 1-20. doi: 10.21892/9789804270178.1

Registro Único de Víctimas. (2020). Reportes. Recuperado de https:// www.unidadvictimas.gov.co/es/ registro-unico-de-victimas-ruv/37394

Richards, A., Ospina-Duque, J., BarreraValencia, M. A., Escobar-Rincón, J., Ardila-Gutiérrez, M., Metzler, T., \& Marmar, C. (2014). Posttraumatic stress disorder, anxiety and depression symptoms, and psychosocial treatment needs in colombians internally displaced by armed conflict: a mixed-method evaluation. Psychological Trauma: Theory, Research, Practice and Policy, 3(4), 384-393. doi: 10.1037/a0022257
Roberts, B., \& Browne J. (2011). A systematic review of factors influencing the psychological health of conflict-affected populations in low and middle-income countries. Global Public Health, 6(8), 814829. doi: 10.1080/17441692.2010.511625

Rojas-Campos, M. (2016). Trastorno de Estrés Postraumático. Revista Médica de Costa Rica y Centroamérica LXXIII, 73(619), 233-240. Recuperado de https://www. medigraphic.com/pdfs/revmedcoscen/ rmc-2016/rmc162h.pdf

Shalev, A., Liberzon, I., \& Marmar, C. (2017). Post-traumatic stress disorder. New England Journal of Medicine, 376(25), 2459-2469. doi: 10.1056/NEJMra1612499

Shultz, J. M., Garfin, D. R., Espinel, Z., Araya, R., Oquendo, M. A., Wainberg, M. L., ... \& Wilson, F. E. (2014). Internally displaced "victims of armed conflict" in Colombia: The trajectory and trauma signature of forced migration. Current Psychiatry Reports, 16(10), 475-491. doi: 10.1007/ s11920-014-0475-7

Torres-Salazar, Y.M., Mejía-Jaimes, L., Conde-Cotes, C.A. \& Botelho-De Oliveira, S. (2021). Víctimas del desplazamiento forzado: comorbilidad entre trastorno por estrés postraumático (TEPT) y depresivo mayor (TDM). Informes Psicológicos, 21(1), 133-149. doi: 10.18566/infpsic.v21n1a09

Venegas-Luque, R., Gutiérrez-Velasco, A., \& Calcedo-Cardeñosa, M. F. (2017). Investigaciones y comprensiones del conflicto armado en Colombia. Salud mental y familia. Universitas Psychologica, 16(3), 1-10. doi: 10.11144/Javeriana. upsy16-3.icca 
Weathers, F. W., Litz, B. T., Herman, D. S., Huska, J. A., \& Keane, T. M. (1993). The PTSD Checklist (PCL): Reliability, validity, and diagnostic utility. In Annual convention of the international society for traumatic stress studies. San Antonio: International Society for Traumatic Stress Studies.
Yehuda, R., Hoge, C. W., McFarlane, A. C., Vermetten, E., Lanius, R. A., Nievergeit, C. M.,... Hyman, S. E. (2015). Posttraumatic stress disorder. Nature Reviews. Disease primers, 1(1), 1-22. doi: 10.1038/ nrdp.2015.57. 